\title{
Impact of long-term medical conditions on the outcomes of psychological therapy for depression and anxiety
}

\author{
Jaime Delgadillo, Alexander Dawson, Simon Gilbody and Jan R. Böhnke
}

\section{Background}

Long-term conditions often coexist with depression and anxiety.

\section{Aims}

To assess the effectiveness of stepped-care psychological therapies for patients with long-term conditions.

\section{Method}

Data from 28498 patients were analysed using regression to model depression (Patient Health Questionnaire (PHQ-9)) and anxiety (Generalised Anxiety Disorder scale (GAD-7)) outcomes. Post-treatment symptoms and effect sizes (d) were estimated for individuals with and without long-term conditions, controlling for covariates. The likelihood of access and response to intensive psychological interventions was also examined.

\section{Results}

Higher post-treatment symptoms were predicted for patients with musculoskeletal problems $(d=0.22-0.27)$, chronic obstructive pulmonary disease $(d=0.26-0.33)$, diabetes $(d=0.05-0.13)$ and psychotic disorders $(d=0.50$ 0.58). Most long-term conditions were associated with greater odds of accessing high-intensity therapies, yet individuals who accessed these continued to have higher average post-treatment symptoms.

\section{Conclusions}

Some long-term conditions are associated with greater intensity of care and poorer outcomes after therapy.

\section{Declaration of interest}

None.

\section{Copyright and usage}

(c) The Royal College of Psychiatrists 2017.
Long-term conditions are highly prevalent in the general population affecting approximately $20 \%$ of people ${ }^{1}$ above the age of 16 and $58 \%$ of people over the age of $60 .^{2}$ Among the more prevalent long-term conditions in the general population are hypertension, chronic pain, gastrointestinal disorders, asthma, arthritis, diabetes, heart disease and chronic obstructive pulmonary disease (COPD). ${ }^{1,3}$ Multimorbidity is common ${ }^{3}$ and these long-term conditions carry a huge financial cost to health services, accounting for approximately $70 \%$ of total health and social care expenditure in public healthcare systems like the UK National Health Service. ${ }^{2}$ This highlights the need to treat both long-term conditions and mental health problems concurrently. Although access to psychological care has been recommended as part of integrated care for people with long-term conditions, ${ }^{4}$ it is possible that the effect of psychological interventions for mental health problems may be attenuated by long-term conditions. For example, Dickens and collaborators ${ }^{5}$ reviewed the efficacy of psychological interventions for depression in patients with coronary heart disease and concluded that clinical trials show mixed evidence with small effect sizes favouring cognitive-behavioural therapy (CBT) as the treatment of choice for this population. Similar conclusions were reached in a systematic review of psychological interventions for persistent pain, which indicates modest effects for CBT in depression and anxiety symptom reductions. ${ }^{6}$ Clinical trials reviewed in these meta-analyses often include participants with highly disabling long-term conditions treated in specialist services or hospital settings, and therefore it is unclear whether these findings are reflective of outcomes from routinely delivered therapy in primary care settings, where the majority of patients' long-term conditions are treated. The objective of this study was to investigate the clinical effectiveness of primary care psychological interventions for depression and anxiety in a large naturalistic sample, comparing outcomes between patients with and without long-term conditions.

\section{Method}

\section{Setting and interventions}

Retrospective clinical case records for a cohort of patients receiving psychological therapy were analysed. These data were gathered as part of routine clinical care at a psychological therapy service in the north of England that was linked to the national Improving Access to Psychological Therapies (IAPT) programme. ${ }^{7}$ The study was conducted as a service evaluation using fully de-identified data, and therefore did not require ethical approval. Patients provided informed consent for their anonymous data to be used for audit, evaluation and research purposes. Patients accessing the service presented with depression- and anxiety-related problems and received evidence-based interventions organised in a stepped-care model. ${ }^{8}$ The majority accessed brief (less than eight sessions), low-intensity guided self-help interventions based on principles of CBT. Those with enduring symptoms after guided self-help and those with more severe conditions had access to high-intensity (up to 20 sessions) therapies including CBT, interpersonal psychotherapy, counselling for depression and eye movement desensitisation and reprocessing (EMDR) for post-traumatic stress.

\section{Measures and data sources}

Consistent with the national IAPT programme, patients in the sample were asked to self-complete three standard outcome measures to monitor progress on a weekly basis. The Patient Health Questionnaire (PHQ-9) is a nine-item measure of major depression symptoms. ${ }^{9}$ Each item is rated on four ordinal response options ( 0 , not at all; 3 , nearly every day), resulting in a depression severity score between 0 and 27 . A cut-off $\geqslant 10$ is 
used to detect clinically significant depression symptoms. The Generalised Anxiety Disorder scale (GAD-7) is a seven-item measure of anxiety symptoms; it is also rated using four ordinal response options ( 0 , not at all; 3 , nearly every day), resulting in an anxiety severity score between 0 and $21 .{ }^{10} \mathrm{~A}$ cut-off score $\geqslant 8$ is recommended to identify the likely presence of a diagnosable anxiety disorder. The Work and Social Adjustment Scale (WSAS) is a measure of functioning across five domains: work, home management, social leisure activities, private leisure activities, and family and relationships. ${ }^{11}$ Each item is rated between 0 (no impairment) and 8 (very severe impairment), with a total severity score between 0 and 40 .

De-identified clinical assessment records were also collected for participants, including demographic (age, gender, ethnicity, employment, socioeconomic deprivation) and clinical information (primary diagnosis, baseline severity, use of psychotropic medication, number of treatment contacts, number of referrals into the service for each patient over a 5-year period, pre- and post-treatment outcome measures described above). Self-reported long-term conditions were gathered using a standardised checklist of chronic illnesses at the time of initial assessment. ${ }^{12}$ This checklist prompts clinicians to gather information about 15 specific conditions including severe (psychotic) mental health problems and an option to note 'other' unspecified conditions. Socioeconomic deprivation was assessed by matching participants' home postcodes to the English Index of Multiple Deprivation $(\mathrm{IMD})^{13}$ and categorising participants into quintile levels of deprivation (categorical variable named IMD).

\section{Sample characteristics}

A total of 32734 case records were gathered for patients who accessed psychological treatment (at least one session) over a period of 5 years between 2010 and 2015. Of these, 2676 (8.2\%) were excluded because they had no recorded information about long-term conditions, and a further $1560(4.8 \%)$ were excluded because no baseline and end scores were available for at least one of the outcome measures (PHQ-9 or GAD-7). This resulted in a sample of 28498 case records that were available for analysis. The mean age in the sample was 38.27 (s.d. =13.94, range 16-92); $64.6 \%$ were female; $85.5 \%$ were of a White British background;

\begin{tabular}{|c|c|c|}
\hline Description & $n$ & $\%$ \\
\hline \multicolumn{3}{|l|}{ Recorded presenting problem $(n=22609)$} \\
\hline Recurrent depression & 8698 & 38.5 \\
\hline Mixed anxiety and depression & 5887 & 26.0 \\
\hline Generalised anxiety disorder & 2725 & 12.1 \\
\hline Depressive episode & 1160 & 5.1 \\
\hline Panic disorder & 970 & 4.3 \\
\hline Social phobia & 540 & 2.4 \\
\hline Obsessive-compulsive disorder & 643 & 2.8 \\
\hline Post-traumatic stress disorder & 565 & 2.5 \\
\hline Specific phobia & 290 & 1.3 \\
\hline Bereavement & 201 & 0.9 \\
\hline Eating disorder & 177 & 0.8 \\
\hline Agoraphobia & 163 & 0.7 \\
\hline Somatoform disorder & 149 & 0.7 \\
\hline Alcohol related mental or behavioural disorder & 40 & 0.2 \\
\hline Bipolar affective disorder & 22 & 0.1 \\
\hline $\begin{array}{l}\text { Does not meet diagnostic criteria for a } \\
\text { common mental disorder }\end{array}$ & 379 & 1.7 \\
\hline Presenting problem not specified in records & 5889 & 20.7 \\
\hline
\end{tabular}

and $36.6 \%$ were unemployed. Primary presenting problems recorded in clinical assessments are presented in Table 1; the most common were recurrent depression $(38.5 \%)$, mixed anxiety and depression (26.0\%) and generalised anxiety disorder (12.1\%). Self-reported long-term conditions are listed in order of prevalence in Table 2. Overall, 23.2\% of patients reported having at least one long-term condition; the most common were asthma $(6.8 \%)$, musculoskeletal problems (chronic pain, $1.8 \%$ ) and hypertension (1.7\%). Approximately $68.0 \%$ of patients in this sample only received low-intensity guided self-help interventions, and $32.0 \%$ accessed high-intensity therapies.

\section{Statistical analysis}

The goals of the analysis were to predict depression (PHQ-9) and anxiety (GAD-7) symptom severity at the end of treatment, controlling for the demographic and clinical characteristics described above, and to compare these outcomes between individuals with and without long-term conditions. Given the typically high correlations between the PHQ-9 and GAD-7 scales, ${ }^{14}$ we applied a seemingly unrelated regression (SUR) model. ${ }^{15}$ SUR models estimate several equations simultaneously when the error terms of these equations are potentially correlated, which is a likely scenario for the prediction equations from PHQ-9 and GAD-7 scores. These models have been applied previously to model correlated dimensions of patient-reported outcomes and have been shown to increase the efficiency of estimates in such situations. ${ }^{16}$ As a sensitivity analysis, we repeated the SUR analysis using a dichotomous variable denoting the presence or absence of a long-term condition, instead of entering dummy variables for the different long-term condition categories. In order to compare outcomes between patients with and without long-term conditions, we estimated effect sizes (Cohen's $d$ ) based on comparing post-treatment outcomes between specific longterm condition groups $v$. the 'no long-term conditions' category and the root mean square error (RMSE) of the respective part (i.e. PHQ-9 or GAD-7) of the SUR model.

As a sensitivity analysis, we assessed differential item functioning (DIF) to verify whether responses to the PHQ-9 and GAD-7 measures are comparable across long-term condition groups, or whether differences in outcome scores could be biased in favour, or against, certain long-term conditions. Specifically, we aimed to assess whether responses to individual PHQ-9 or GAD-7 items corresponded to the same psychopathology severity levels across long-term condition groups. To achieve this, we applied logistic

\section{Table 2 Self-reported long-term medical conditions $(n=28498)$}

\begin{tabular}{lrr} 
Description & $n$ & $\%$ \\
None & 21882 & 76.8 \\
Other (unspecified) & 2316 & 8.1 \\
Asthma & 1935 & 6.8 \\
Chronic musculoskeletal & 507 & 1.8 \\
Hypertension & 490 & 1.7 \\
Non-insulin-dependent diabetes mellitus & 327 & 1.1 \\
Epilepsy & 188 & 0.7 \\
Chronic obstructive pulmonary disease & 137 & 0.5 \\
Coronary heart disease & 137 & 0.5 \\
Severe (psychotic) mental health problems & 134 & 0.5 \\
Insulin-dependent diabetes mellitus & 111 & 0.4 \\
Cancer & 114 & 0.4 \\
Stroke and transient ischaemic attack & 76 & 0.3 \\
Multiple sclerosis & 49 & 0.2 \\
Chronic kidney disease & 41 & 0.1 \\
Heart failure & 38 & 0.1 \\
Parkinson's disease & 16 & 0.1 \\
\hline
\end{tabular}


ordinal regressions standardising on latent variable estimates of depression and anxiety using a Generalised Partial Credit Model (uniform and non-uniform DIF), as described by Crane et al. ${ }^{17}$ We evaluated differences in Pseudo- $R^{2}$ values between regression models to assess whether the long-term condition categories explained a relevant amount of variance in final PHQ-9 and GAD-7 scores.

Considering the stepped-care context in which psychological interventions were provided to this sample, we carried out secondary analyses to investigate whether patients with long-term conditions may differ in their probability of receiving and responding to high-intensity therapy, which would be plausible given their general higher level of distress and functional impairment. To test this hypothesis, we used the same predictors described above (in the SUR model) in a logistic regression model aiming to predict the likelihood of concluding a treatment episode at the higher step of care (high-intensity therapy $v$. low-intensity care as a reference category). In this analysis $n=29(0.1 \%$ of total $)$ case records were excluded because of inputting errors in the clinical database, which did not enable us to identify the assignment of individuals to low- or high-intensity steps of care. Furthermore, we repeated the SUR analysis separately analysing individuals that finished their treatment episode after accessing low- $(n=18902)$ or high- $(n=8884)$ intensity interventions.

Since the amount of missing data was only minimal $\left(n_{\text {miss }}=683,2.4 \%\right.$ of available cases $)$, no additional imputation analyses were undertaken. As expected, most missing data points were for the outcome measures (PHQ-9 and GAD-7) and 390 individuals did not have valid postcodes documented in clinical records, so IMD could not be derived. Furthermore, IMD data could not be imputed, since it is a geographical rather than an individual characteristic.

\section{Results}

\section{SUR equations modelling}

As expected, the equations for PHQ-9 and GAD-7 outcomes were highly correlated. The correlation between the error terms of the two equations was large $(r=0.82)$ and statistically significant (Breusch-Pagan test, $\chi^{2}=18704.17, P<0.001$ ). The SUR model explained a moderate amount of variance in post-treatment outcomes (PHQ-9 34\%; GAD-7 29\%). Table 3 presents the SUR model results in the full sample, which are interpreted below. In what follows, we refer to combined depression (PHQ-9) and anxiety (GAD-7) symptoms at the time of the last attended treatment session as 'post-treatment distress levels'.

\section{Analysis of demographic variables}

Age was significantly correlated with both outcomes, although the association was weak, equivalent to a reduction of 0.01 score points per year increase. Gender did not correlate with either measure. In contrast, unemployment was associated with higher average post-treatment distress; with an increase of 0.68 points for PHQ-9 and 0.54 points for GAD-7. Patients from South Asian backgrounds, dual heritage and other ethnicities also had higher average post-treatment distress in comparison with patients from White British backgrounds. The IMD quintiles also show significant associations in the expected direction: patients living in more socioeconomically deprived areas (as defined by their home postcode) tended to have greater symptom severity at the end of treatment, and there was an increasing trend in mean post-treatment scores for each quintile of deprivation.
Table 3 Estimated coefficients for the seemingly unrelated regression (SUR) model jointly predicting post-treatment depression (PHQ-9) and anxiety (GAD-7) severity

\begin{tabular}{|lcc|}
\multirow{2}{*}{ Variable } & \multicolumn{2}{c|}{$B$ (s.e.) } \\
\cline { 2 - 3 } & PHQ-9 & GAD-7 \\
\hline PHQ-9 (baseline severity) & $0.31^{* * *}(0.00)$ & - \\
\hline GAD-7 (baseline severity) & - & $0.33^{* * *}(0.00)$ \\
\hline WSAS (baseline severity) & $0.17^{* * *}(0.00)$ & $0.13^{* * *}(0.00)$ \\
\hline Age (years) & $-0.01^{* *}(0.00)$ & $-0.01^{* * *}(0.00)$ \\
\hline Female & $-0.07(0.07)$ & $0.02(0.07)$ \\
\hline Unemployed & $0.68^{* * *}(0.07)$ & $0.54^{* * *}(0.07)$ \\
\hline
\end{tabular}

Ethnicity (reference category

White British)

Dual heritage $\quad 0.49 *(0.23) \quad 0.56^{* *}(0.20)$

South Asian $\quad 1.29^{* * *}(0.18) \quad 1.13^{* * *}(0.16)$

Black British, African or Caribbean $\quad-0.37(0.25) \quad-0.38(0.22)$

Other $\quad 0.53^{* * *}(0.15) \quad 0.45^{* * *}(0.13)$

IMD (reference category

1st IMD quintile)

2nd quintile $\quad-0.77^{* * *}(0.11)-0.63^{* * *}(0.10)$

3rd quintile $\quad-1.11^{* * *}(0.11)-0.85^{* * *}(0.09)$

4th quintile $\quad-1.36^{* * *}(0.11)-1.03^{* * *}(0.10)$

5th quintile $\quad-1.75^{* * *}(0.12)-1.33^{* * *}(0.10)$

Medication $\quad-0.30(0.18) \quad-0.20(0.16)$

Number of referrals into service $\quad 0.59^{* * *}(0.03) \quad 0.55^{* * *}(0.03)$

Treatment contacts attended $\quad-0.25^{* * *}(0.01)-0.22^{\star * *}(0.01)$

Mental health (reference

category depressive episode)

Recurrent depression

Mixed anxiety and depression

Generalised anxiety disorder

Social phobia

Panic disorder

Agoraphobia

Specific phobia

OCD

PTSD

Bereavement

Eating disorder

Somatoform disorder

Does not meet CMD criteria

Other diagnosis

$\begin{array}{ll}0.36(0.19) & 0.29(0.17) \\ 0.16(0.10) & 0.22^{*}(0.09) \\ -0.56^{* * *}(0.14) & -0.14(0.12) \\ -0.30(0.27) & -0.24(0.24) \\ -0.43^{*}(0.20) & -0.12(0.18) \\ -0.09(0.46) & 0.15(0.41) \\ -0.23(0.36) & 0.10(0.31) \\ -0.25(0.25) & 0.69^{* *}(0.22) \\ 0.55^{*}(0.26) & 1.01^{* * *}(0.23) \\ 0.76(0.42) & 0.69(0.38) \\ 1.64^{* * *}(0.45) & 0.71(0.40) \\ -1.18^{*}(0.49) & -0.66(0.43) \\ 0.38(0.31) & 0.18(0.27) \\ 0.06(0.11) & 0.16(0.10)\end{array}$

LTC (reference category

no self-reported LTC)

$\begin{array}{lll}\text { Asthma } & 0.17(0.14) & 0.21(0.12) \\ \text { Cancer } & 0.48(0.55) & 0.03(0.48)\end{array}$

$\begin{array}{lll}\text { Chronic musculoskeletal } & 1.56^{* * *}(0.26) & 1.15^{* * *}(0.23)\end{array}$

COPD

Cardiovascular

Diabetes

Epilepsy

Severe mental health problems

Other LTC

$1.92^{* * *}(0.51) \quad 1.35^{* *}(0.45)$

$0.10(0.22) \quad-0.05(0.20)$

$0.77^{* *}(0.29) \quad 0.24(0.25)$

$-0.07(0.43) \quad 0.04(0.38)$

$3.36^{* * *}(0.51) \quad 2.58^{* * *}(0.45)$

Constant

\begin{tabular}{ll}
$0.67 * * *(0.13)$ & $0.52 * * *(0.11)$ \\
\hline $3.74 * * *(0.27)$ & $3.15^{* * *}(0.24)$
\end{tabular}

Observations

$R$-squared

27815

27815
IMD ind Disorder (anxiety measure); WSAS, Work and Social Adjustment Scale;

disordex of multiple deprivation (quintile groups); $O C D$, obsessive-compulsive disorder; PTSD, post-traumatic stress disorder; CMD, common mental disorder; LTC, long-term condition; COPD, chronic obstructive pulmonary disease.

${ }^{*} P<0.05 ;{ }^{* *} P<0.01 ;{ }^{* *} P<0.001$.

\section{Analysis of treatment-related variables}

Differences attributable to taking medication were small and not statistically significant. The number of referrals for psychological treatment (over the past 5 years) was significantly associated with post-treatment outcomes. Each additional referral for care predicted an increase of 0.59 points for PHQ-9 and 0.55 for 
GAD-7. Since up to 11 referrals were observed in the data-set, this could account for substantially greater post-treatment symptom severity for patients with multiple treatment episodes. A greater number of treatment contacts (in the index treatment episode) was associated with lower average post-treatment distress; decreasing this by -0.25 PHQ-9 points per contact attended ( -0.22 for GAD-7).

Some differences between diagnostic categories were observed. A patient with a diagnosis of depression (the reference category) will finish therapy with a predicted PHQ-9 score of 9.69 (s.e. $=0.08$, marginal mean) and GAD-7 score of 8.45 (s.e. $=0.07$ ). Some diagnoses were associated with higher post-treatment distress in comparison with the above reference scores; these were obsessive-compulsive disorder (GAD-7), post-traumatic stress disorder (PHQ-9 and GAD-7) and eating disorders (PHQ-9). Diagnoses associated with lower predicted distress levels compared with depression were generalised anxiety disorder (PHQ-9), panic disorder (PHQ-9) and somatoform disorders (PHQ-9). As expected, baseline severity (PHQ-9, GAD-7) and functional impairment (WSAS) measures were significantly correlated with post-treatment outcomes, such that individuals who were more severely impaired were expected to have higher post-treatment distress severity.

\section{Analysis of long-term conditions data}

After controlling for all of the above demographic and clinically relevant variables, five long-term condition categories were associated with higher post-treatment distress levels: chronic musculoskeletal problems, COPD, severe mental health problems, diabetes, and 'other' non-specified conditions. Figure 1 presents predicted marginal post-treatment outcome scores and Sidakadjusted 95\% confidence intervals; this shows that estimated post-treatment means differed substantially across the categories of long-term conditions. The figure also presents the estimated treatment effect sizes corresponding to each long-term condition category, by comparison with the reference group without any self-reported long-term conditions (and in relation to nonexplained variance in the regression model; $R M S E_{\mathrm{PHQ}-9}=5.82$;

(a)

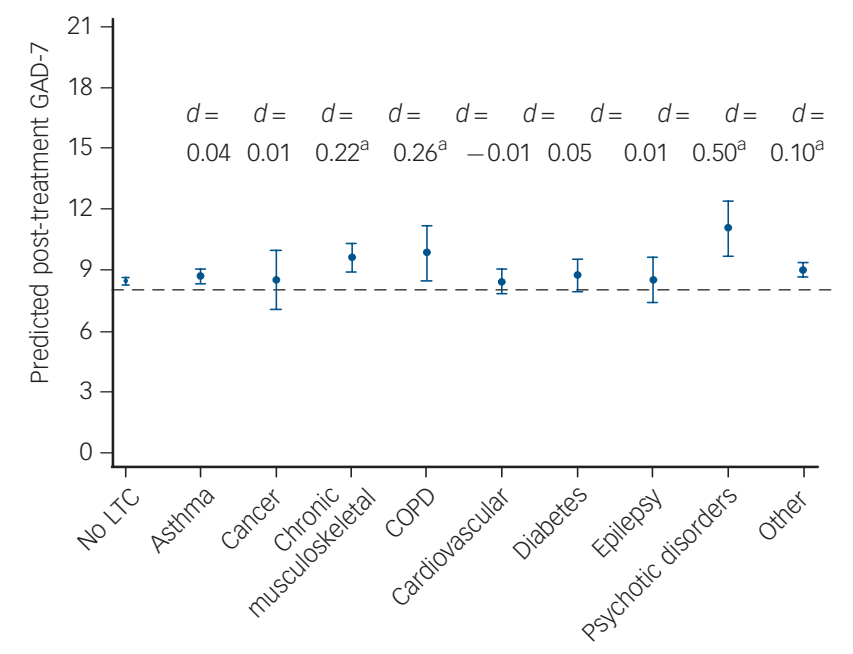

$\left.R M S E_{\mathrm{GAD}-7}=5.14\right)$. The effect sizes for those five long-term condition categories, associated with poorer outcomes, range from small (around $d=0.20$ ) to medium (around 0.50; according to Cohen $\left.{ }^{18}\right)$.

\section{Sensitivity and secondary analyses}

Our sensitivity analyses found no evidence of DIF on PHQ-9 or GAD-7 across long-term condition groups. The maximal difference in Pseudo- $R^{2}$ values across regression models was 0.001 , which is far smaller than the recommended cut-off of $0.035,{ }^{17}$ indicating that only very small amounts of variance in item responses were related to specific long-term conditions (online supplement DS1 and Tables DS1-DS8).

The SUR model including a dichotomous variable (long-term condition $v$. no long-term condition) resulted in an adjusted mean difference of 0.57 (s.e. $=0.09, P<0.001$ ) for the PHQ-9 and 0.42 for GAD-7 (s.e. $=0.08, P<0.001$ ), which speaks for a general disadvantage of this population with long-term conditions after controlling for potentially confounding variables. However, this effect is rather small $\left(E S_{\mathrm{PHQ}}=0.10\right.$ and $E S_{\mathrm{GAD}}=0.08$; coefficients standardised on regression RMSE) and therefore modelling posttreatment outcomes for each specific long-term condition is more informative. When we repeated the SUR analysis in the separate samples of individuals that finished treatment after low- or high-intensity interventions, the results (online supplement DS2 and Table DS9) were largely consistent with the main SUR model shown in Table 3 . The only differences were found for people with COPD and diabetes. COPD continued to be associated with higher post-treatment PHQ-9 $(B=3.81, P<0.001)$ and GAD-7 $(B=2.34, P<0.01)$ scores after high-intensity interventions, but not after low-intensity ones (PHQ-9: $B=0.90, P>0.05$; GAD-7: $B=0.75, P>0.05)$. Diabetes continued to be associated with higher post-treatment PHQ-9 $(B=1.30, P<0.01)$ but not with GAD-7 $(B=0.50, P>0.05)$ scores after high-intensity interventions; no such associations were found for individuals that only accessed low-intensity interventions (PHQ-9: $B=0.43, P>0.05$; GAD-7: $B=0.00, P>0.05)$. 
Table 4 Probability of accessing high-intensity therapy for patients with long-term conditions ${ }^{\text {a }}$

Long-term condition Odds ratio $(95 \% \mathrm{Cl})$

Asthma

Cancer

Chronic musculoskeletal

Chronic obstructive pulmonary disease Cardiovascular

Diabetes

Epilepsy

Severe (psychotic) mental health problems

Other non-specified long-term condition

$1.23^{* *}(1.09-1.39)$

$1.66 *(1.03-2.65)$

$1.47^{* *}(1.17-1.84)$

$1.60 *(1.04-2.47)$

$1.15(0.94-1.40)$

$1.39 *(1.07-1.79)$

$0.99(0.68-1.44)$

$1.00(0.64-1.57)$

$1.30 * * *(1.17-1.46)$

a. All coefficients controlled for demographic and treatment-related variables as for the main analysis (see Table 3).

${ }^{\star} P<0.05 ;{ }^{* *} P<0.01 ;{ }^{* * *} P<0.001$

Table 4 shows the results of the logistic regression model predicting the likelihood of receiving high-intensity therapy. As expected, patients with most long-term conditions had a significantly higher probability of receiving high-intensity interventions (odds ratios $(\mathrm{OR})=1.23-1.66$; all $P<0.05$ ). The only exceptions to this were found for patients with cardiovascular conditions, epilepsy and severe (psychotic) mental disorders, who were no more likely to receive high-intensity therapy in comparison with those without any self-reported long-term conditions.

\section{Discussion}

The overall goal of this study was to compare the effects of routinely delivered psychological care for patients with and without self-reported long-term conditions. We found that patients with certain long-term conditions tend to finish psychological treatment with greater depression and anxiety severity. In comparison with patients without long-term conditions, this trend was statistically significant for musculoskeletal problems (effect sizes $d=0.22-0.27)$, COPD ( $d=0.26-0.33$ ), diabetes $(d=0.05-0.13)$, severe mental health problems $(d=0.50-0.58)$ and 'other' non-specified conditions $(d=0.10$ $0.11)$. These findings converge with some observations of small to moderate effect sizes reported by meta-analyses of psychological interventions. $^{6,19-21}$ Furthermore, patients with most types of self-reported long-term conditions (except cardiovascular conditions, epilepsy and severe mental disorders) were significantly more likely to receive more intensive and costly psychological interventions, consistent with their higher level of impairment and symptom severity. Accessing high-intensity therapy continued to be associated with higher average post-treatment distress in secondary analyses, indicating that patients with long-term conditions are not necessarily better off after highintensity ( $v$. low-intensity) care in this primary care setting. We noted that, in particular, severe mental health problems (such as psychotic disorders) tended to be strongly associated with poorer outcomes, although the prevalence of such conditions was very small $(0.5 \%)$ given the service's remit to offer treatment for common mental health problems. This evidence may indicate that the intensity and type of interventions offered in this primary care setting are clearly inadequate to improve psychological distress symptoms in people with a history of severe and enduring (i.e. psychotic) mental disorders. It is also plausible that those with severe mental disorders were identified as such during the early phases of low-intensity interventions and appropriately referred onwards to secondary care/psychiatric services; hence explaining why these individuals were not more likely to access high-intensity therapies in this primary care setting.

A range of other demographic and clinical factors were also associated with post-treatment outcomes. Baseline severity and impairment measures, employment status, socioeconomic deprivation and age have been shown to predict outcomes in comparable clinical samples and settings. ${ }^{22-24}$ In addition, the present results also indicate that patients from certain ethnic backgrounds (South Asian, dual heritage) and diagnostic groups (obsessive-compulsive disorder, post-traumatic stress disorder and eating disorders) may be at increased risk of poor treatment outcomes.

The overall prevalence of self-reported long-term conditions in this cohort of patients receiving psychological treatment (23.2\%) was comparable with general adult population estimates (20\%) from England. ${ }^{1}$ Asthma was the most prevalent (identifiable) long-term condition (around $6 \%$ of patients); which may reflect the common coexistence of this condition with anxiety-related problems. However, a comparison of specific categories reveals a disproportionately small representation of certain conditions such as hypertension $(1.7 \%$ in our sample $v .14 .3 \%$ in the general population), musculoskeletal problems (1.8\% v. 14.0\%), diabetes $(1.5 \%$ v. $3.8 \%)$, heart failure $(0.1 \% v .2 .0 \%)$ and COPD $(0.5 \% v$. $1.8 \%)$. These discrepancies possibly indicate that patients with certain conditions are much less likely to access psychological care; for example, the large discrepancy for hypertension indicates a ratio of 1:12. This discrepancy might in some cases be explained by the existence of specialist teams for people with long-term conditions; for example, the local area for this cohort of patients had two specialist musculoskeletal treatment services. However, it is possible that some patients with long-term conditions commonly treated in primary care clinics may be inadequately screened or seldom referred for mental health treatment. Recent research indicates that this may be the case for some patients with coronary heart disease and diabetes. ${ }^{25}$

\section{Strengths and limitations}

This large ( $n=28498)$ naturalistic cohort was adequately powered to assess the predictive value of multiple demographic and clinical variables, which was optimally modelled using joint-prediction of correlated outcomes (PHQ-9, GAD-7). Our analyses additionally contained another layer of robustness tests. It is important to establish measurement invariance to have confidence that outcome questionnaires can be interpreted similarly across different patient groups. ${ }^{26,27}$ Our sensitivity analyses found no evidence of DIF in PHQ-9 or GAD-7, which indicates that results of these outcome measures have the same meaning across longterm conditions and non-long-term condition groups in this sample. This finding supports the notion that post-treatment outcome differences between long-term condition groups are likely to represent actual differences in psychological distress, rather than measurement error or confounding of long-term condition symptoms with mental health symptoms.

Some limitations to note include the reliance on self-report of long-term conditions, as is common in large cohort and epidemiological studies. ${ }^{1,28}$ In particular, we did not have more specific information about the types of long-term condition of patients who endorsed the 'other' category in assessment records. Presenting problem categories for mental health issues were also likely to be error prone, since these were ascertained using brief screening measures ${ }^{12}$ rather than structured diagnostic interviews. Future studies that gather long-term condition diagnoses recorded in medical records and structured diagnostic interviews may render more precise outcome prediction estimates. A further 
caveat is that the data from this study, albeit large, came from a single site in the north of England. Future replication studies using data from similar stepped-care services in other regions would enable us to assess the extent to which these findings are generalisable.

\section{Implications for clinical practice}

The impact of, and need for, integrated mental health services has often been discussed from a medical perspective, i.e. how psychological professionals could be brought into medical contexts. $^{29,30}$ Specialist medical knowledge about long-term conditions is a key element of success and the integration of medical expertise within mental health services could also help to improve treatment outcomes in these settings. Our observations raise questions about the effectiveness of routinely delivered stepped-care psychological treatments for people with comorbid diabetes, COPD and chronic pain. These results also mirror findings from research into health-related quality of life (QOL), where it is also found that certain long-term conditions (such as chronic pain) and especially having multiple long-term conditions can considerably undermine QOL and exacerbate psychological distress. ${ }^{31}$ Such studies highlight the importance of multidisciplinary care aiming to target multiple facets of well-being, adjustment and QOL. It may be particularly important to offer integrated multidisciplinary care for people with specific conditions described above. For example, collaborative care interventions can enhance self-management of depression symptoms for patients with diabetes and coronary heart disease. ${ }^{29}$ Overall, we conclude that standard stepped-care interventions are insufficient to support patients with multimorbidity, especially if delivered in isolation from other healthcare specialists. Our observations concur with recent calls for closer integration of physical and mental healthcare. ${ }^{32}$

Healthcare economies and policy-makers should systematically investigate the prevalence of long-term conditions in people using mental health services. This information could be crucial to design and deliver more integrated care for patients with long-term conditions, but can also serve to understand how the demographic and clinical profile of local populations could have an impact on service outcomes. This aspect is still underexplored, especially when thinking about new benchmarking models and quality indicators within primary care psychological services. ${ }^{33}$

Jaime Delgadillo, PhD, Leeds Community Healthcare NHS Trust and Department of Health Sciences, University of York, York, UK; Alexander Dawson, Leeds Community Healthcare NHS Trust, Leeds; Simon Gilbody, DPhil, FRCPSych, Jan R. Böhnke, Dipl Psych, Dr rer nat, Hull York Medical School, and Department of Health Sciences, University of York, York, UK.

Correspondence: Jaime Delgadillo, Clinical Psychology Unit, Department of Psychology, University of Sheffield, Western Bank, Sheffield S10 2TN, UK. Email: jaime.delgadillo@nhs.net

First received 8 Jun 2016, final revision 25 Jul 2016, accepted 30 Jul 2016

\section{Funding}

This study was funded by Leeds Community Healthcare NHS Trust and commissioned by the Long Term Conditions Project Team, Leeds, UK, January 2016.

\section{Acknowledgements}

We thank Peter A. Coventry for reviewing an earlier version of the study manuscript and for helpful advice.

\section{References}

1 Weich S, Bebbington P, Rai D, Stranges S, McBride O, Spiers N, et al. The population impact of common mental disorders and long-term physical conditions on disability and hospital admission. Psychol Med 2013; 43: 921-31.
2 Department of Health. Long Term Conditions Compendium of Information (3rd edn). Department of Health, 2012.

3 Barnett K, Mercer SW, Norbury M, Watt G, Wyke S, Guthrie B. Research paper. Epidemiology of multimorbidity and implications for health care, research, and medical education: a cross-sectional study. Lancet 2012; 380 : 37-43.

4 Department of Health. Talking Therapies: A Four-Year Plan of Action. Department of Health, 2011.

5 Dickens C, Cherrington A, Adeyemi I, Roughley K, Bower P, Garrett C, et al. Characteristics of psychological interventions that improve depression in people with coronary heart disease: a systematic review and meta-regression. Psychosom Med 2013; 75: 211-21.

6 Williams ACD, Eccleston CM, Morley S. Psychological therapies for the management of chronic pain (excluding headache) in adults. Cochrane Database System Rev 2012; 11: CD007407.

7 Clark DM, Layard R, Smithies R, Richards DA, Suckling R, Wright B. Improving access to psychological therapy: initial evaluation of two UK demonstration sites. Behav Res Ther 2009; 47: 910-20.

8 National Institute for Health and Care Excellence. Common Mental Health Disorders: Identification and Pathways to Care. CG123. National Institute for Health and Care Excellence, 2011 (http://www.nice.org.uk/guidance/CG123).

9 Kroenke K, Spitzer RL, Williams JB. The PHQ-9: validity of a brief depression severity measure. J Gen Intern Med 2001; 16: 606-13.

10 Kroenke K, Spitzer RL, Williams JBW, Monahan PO, Löwe B. Anxiety disorders in primary care: prevalence, impairment, comorbidity, and detection. Ann Intern Med 2007; 146: 317-25.

11 Mundt JC, Marks IM, Shear MK, Greist JM. The Work and Social Adjustment Scale: a simple measure of impairment in functioning. Br J Psychiatry 2002; 180: 461-4.

12 IAPT National Programme Team. The IAPT Data Handbook: Guidance on Recording and Monitoring Outcomes to Support Local Evidence-Based Practice. Version 2.0.1. National IAPT Programme Team, 2011 (http:// www.iapt.nhs.uk/silo/files/iapt-data-handbook-v2.pdf)

13 Department for Communities and Local Government. The English Indices of Deprivation 2010. Department for Communities and Local Government, 2011.

14 Böhnke JR, Lutz W, Delgadillo J. Negative affectivity as a transdiagnostic factor in patients with common mental disorders. J Affect Disord 2014; 166 270-8.

15 Zellner A. An efficient method of estimating seemingly unrelated regressions and tests for aggregation bias. J Am Stat Assoc 1962; 57: 348-68.

16 Keshavarzi S, Ayatollahi SMT, Zare N, Farkhondeh S. Quality of life of childbearing age women and its associated factors: an application of seemingly unrelated regression (SUR) models. Qual Life Res 2013; 22: 1255-63.

17 Crane PK, Gibbons LE, Ocepek-Welikson K, Cook K, Cella D, Narasimhalu K, et al. A comparison of three sets of criteria for determining the presence of differential item functioning using ordinal logistic regression. Qual Life Res 2007; 16: 69-84.

18 Cohen J. Statistical Power Analysis for the Behavioural Sciences (2nd edn). Erlbaum, 1988.

19 Coventry PA, Gellatly JL. Improving outcomes for COPD patients with mild-to-moderate anxiety and depression: a systematic review of cognitive behavioural therapy. Br J Health Psychol 2008; 13: 381-400.

20 Panagioti M, Scott C, Blakemore A, Coventry PA. Overview of the prevalence, impact, and management of depression and anxiety in chronic obstructive pulmonary disease. Int J Chron Obstruct Pulmon Dis 2014; 9: 1289-306.

21 van der Feltz-Cornelis CM, Nuyen J, Stoop C, Chan J, Jacobson AM, Katon W, et al. Effect of interventions for major depressive disorder and significant depressive symptoms in patients with diabetes mellitus: a systematic review and meta-analysis. Gen Hosp Psychiatry 2010; 32: 380-95.

22 Delgadillo J, Asaria M, Ali S, Gilbody S. On poverty, politics and psychology: the socioeconomic gradient of mental healthcare utilisation and outcomes. Br J Psychiatry 2016; 209: 429-30.

23 Delgadillo J, Moreea O, Lutz W. Different people respond differently to therapy: a demonstration using patient profiling and risk stratification. Behav Res Ther 2016; 79: 15-22.

24 Firth N, Barkham M, Kellett S, Saxon D. Therapist effects and moderators of effectiveness and efficiency in psychological wellbeing practitioners: a multilevel modelling analysis. Behav Res Ther 2015; 69: 54-62.

25 Coventry PA, Hays R, Dickens C, Bundy C, Garrett C, Cherrington A, et al Talking about depression: a qualitative study of barriers to managing depression in people with long term conditions in primary care. BMC Fam pract 2011; 12: 10. 
26 Böhnke JR, Croudace TJ. Factors of psychological distress: clinical value, measurement substance, and methodological artefacts. Soc Psychiatry Psychiatr Epidemiol 2015; 50: 515-24.

27 Teresi JA, Ocepek-Welikson K, Kleinman M, Eimicke JP, Crane PK, Jones RN et al. Analysis of differential item functioning in the depression item bank from the patient reported outcome measurement information system (PROMIS): an item response theory approach. Psychol Sci Q 2009; 51: 148-80

28 Patten SB, Beck CA, Kassam A, Williams JV, Barbui C, Metz LM. Long-term medical conditions and major depression: strength of association for specific conditions in the general population. Can J Psychiatry 2005; 50: 195-202.

29 Coventry P, Lovell K, Dickens C, Bower P, Chew-Graham C, McElvenny D, et al. Integrated primary care for patients with mental and physical multimorbidity: cluster randomised controlled trial of collaborative care for patients with depression comorbid with diabetes or cardiovascular disease. BMJ 2015; 350: h638.

30 McDaniel SH, deGruy III FV. An introduction to primary care and psychology. Am Psychol 2014; 69: 325-31.

31 Tyack Z, Frakes KA, Barnett A, Cornwell P, Kuys S, McPhail S. Predictors of health-related quality of life in people with a complex chronic disease including multimorbidity: a longitudinal cohort study. Qual Life Res 2016; 25: 2579-92.

32 Naylor C, Das P, Ross S, Honeyman M, Thompson J, Gilburt H. Bringing Together Physical and Mental Health: A New Frontier for Integrated Care. King's Fund, 2016.

33 Delgadillo J, McMillan D, Leach C, Lucock M, Gilbody S, Wood N. Benchmarking routine psychological services: a discussion of challenges and methods. Behav Cogn Psychother 2014; 42: 16-30.

\section{poems by doctors}

\section{Research of Hope}

\section{Sally Fox}

My perception of safety has always been skewed

My defensive shyness often construed as rudeness The freezing sensation I feel inside

when introduced to a new person

I'm searching their features for a sense of familiar looking for something kind or similar

But danger is always omnipresent.

It makes me feel awkward and hesitant,

resistant to friendship.

Then at a PD conference Jeremy Hall spoke

of the research he'd done

with Merrick Pope and another.

MRI scans of borderline brains -

their findings seem to proclaim

I'm not a social moron after all;

it's down to that concentration of neurons -

that almond cluster they call the amygdala

in the pre-frontal cortex.

That's the reason for my social awkwardness.

Its hypersensitivity makes so much sense to me -

born of childhood adversity -

confused facial emotion recognition

affecting my social cognition.

This science of magnetic resonance imaging

is so bloody validating!

At long last - some Dignity and Hope.

Thank you: Nicol, Hall and Pope!

Selected by Femi Oyebode. From Stigma \& Stones: Living with a Diagnosis of BPD, poems by Sally Fox \& Jo McFarlane. (c) Sally Fox. Reprinted with permission.

Through their collection Stigma \& Stones, writers/performers/partners Sally Fox and Jo McFarlane seek to promote understanding, improve treatment and reduce the stigma of living with a diagnosis of BPD.

The British Journal of Psychiatry (2017)

210, 53. doi: $10.1192 /$ bjp.bp. 116.181719 